\title{
Development of gated fiber detectors for laser-induced strong electromagnetic pulse environments
}

\author{
Po Hu${ }^{1,2,3,4} \cdot$ Zhi-Guo $\mathrm{Ma}^{4} \cdot \mathrm{Kai} \mathrm{Zhao}^{4} \cdot$ Guo-Qiang Zhang ${ }^{5} \cdot$ De-Qing Fang ${ }^{4}$. \\ Bao-Ren $\mathrm{Wei}^{4} \cdot$ Chang-Bo $\mathrm{Fu}^{4}{ }^{4} \cdot \mathrm{Yu}^{4}-\mathrm{Gang} \mathrm{Ma}^{2,4}$
}

Received: 9 February 2021/Revised: 22 April 2021 / Accepted: 29 April 2021 / Published online: 9 June 2021

(C) The Author(s) 2021

\begin{abstract}
With the development of laser technologies, nuclear reactions can happen in high-temperature plasma environments induced by lasers and have attracted a lot of attention from different physical disciplines. However, studies on nuclear reactions in plasma are still limited by detecting technologies. This is mainly due to the fact that extremely high electromagnetic pulses (EMPs) can also be induced when high-intensity lasers hit targets to induce plasma, and then cause dysfunction of many types of traditional detectors. Therefore, new particle detecting technologies are highly needed. In this paper, we report a recently developed gated fiber detector which can be used in harsh EMP environments. In this prototype detector, scintillating photons are coupled by fiber and then
\end{abstract}

This work was supported by the National Nature Science Foundation of China (Nos. 11875191, 11890714, 11925502, 11935001, and 11961141003) and the Strategic Priority Research Program (No. CAS XDB1602).

Chang-Bo Fu

cbfu@fudan.edu.cn

Yu-Gang Ma

mayugang@fudan.edu.cn

1 Shanghai Institute of Applied Physics, Chinese Academy of Sciences, Shanghai 201800, China

2 School of Physical Science and Technology, Shanghai Tech University, Shanghai 201210, China

3 School of Nuclear Sciences and Technology, University of Chinese Academy of Sciences, Beijing 100049, China

4 Key Laboratory of Nuclear Physics and Ion-beam Application (MOE), Institute of Modern Physics, Fudan University, Shanghai 200433, China

5 Shanghai Advanced Research Institute, Chinese Academy of Sciences, Shanghai 201210, China transferred to a gated photomultiplier tube which is located far away from the EMP source and shielded well. With those measures, the EMPs can be avoided which may result that the device has the capability to identify a single event of nuclear reaction products generated in laser-induced plasma from noise EMP backgrounds. This new type of detector can be widely used as a time-of-flight (TOF) detector in high-intensity laser nuclear physics experiments for detecting neutrons, photons, and other charged particles.

Keywords Gated fiber detector - Radiation detection . High-intensity laser $\cdot$ Strong electromagnetic pulses

\section{Introduction}

With the development of high-intensity laser (HIL) technologies, it is possible today to create plasma environments for fundamental nuclear studies or nuclear applications [1-10]. For example, in inertial confinement fusion experiments, multiple ns-pulse-width HILs can compress targets which are composed of deuterium or tritium to densities as high as 10,000 times of theirs initial [11], and then igniting nuclear reactions. High-energy neutrons and protons can be produced in this process through reactions $\mathrm{D}(\mathrm{D}, \mathrm{n})^{3} \mathrm{He}, \mathrm{D}(\mathrm{D}, \mathrm{p})^{3} \mathrm{H}$, or $\mathrm{D}(\mathrm{T}, \mathrm{n})^{4} \mathrm{He}$, etc. In laser Coulomb explosion experiments [12, 13], a fspulse-width HIL hits on deuterium nano-clusters, strips their electrons, and then cause coulomb explosion of the deuterium ions. Nuclear reactions are triggered when D ions start colliding with each other. Nuclear reactions have also been observed in the so-called laser plasma collider scheme $[3,6,14]$, where plasmas induced by ns-pulse- 
width HILs collide with each other head on head. With more and more HIL facilities running or under construction, this new interdisciplinary, so-called laser nuclear physics, will have a brilliant future.

However, methods of detecting nuclear products induced by HILs are still limited and needed urgently. In over 100 years of nuclear and particles physics history, various types of detectors have been developed for different environments, such as scintillating photon-based detectors (e.g., plastic, liquid, and gas scintillator), semiconductor-based detectors (e.g., Si, high-purity germanium, and diamond), and traced detectors (for example, CR39) [15, 16]. However, most of these detection technologies cannot be used directly in HIL environments because of the following difficulties.

In a typical high laser experimental environment, electromagnetic pulses (EMPs) [7, 17, 18] can interfere with many types of traditional detectors, and cause them dysfunction. When a high-intensity laser focuses on a target, it interacts with the target's materials and causes the emission of photons (or, in other words, electromagnetic waves) at almost any frequency. The photon spectra could cover radio frequency, microwave, infrared, optical, X-rays, as well as $\gamma$-ray domains. For semiconductors, bias voltages are needed when running them. However, in high-EMP environments, the electromagnetic fields of the EMP can be much larger than applied bias voltages (fields), causing errors or even damage to the detectors. The same problems appear in photomultiplier tubes (PMTs) too. EMP fields could highly distort the fields applied between a PMT's dynodes and then cause its dysfunction. Therefore, even scintillators themselves may also work under EMPs, but due to the dysfunction of PMTs coupled to them, traditional setups still cannot work in strong EMP environments. EMPs can also cause dysfunction of electronics, including amplifiers, amplitude-to-digital converters (ADCs), and computers. Particularly, strong microwaves generated during laser-target interactions are recognized as a threat to electronics and computers [19].

Trace detectors like CR39 [20-23] or Thomson spectrometers which record particles with image plates [24, 25], etc. are not sensitive to the EMPs. Therefore, they have been widely used in HIL experiments today. However, their detecting sensitivities are very limited, and they are not so convenient to be used too. Detecting nuclear reaction products down to a single particle in an HIL environment is still challenging. The development of new robust radiation detectors is very important for further progress in laser nuclear physics.

In this paper, we present a recently developed gated fiber detector (GFD) that can be used in strong electromagnetic environments [26]. In the second section, the structure of the GFD will be described, and in the third section, online testing results will be given, followed by a summary.

\section{Structure of the gated fiber detector}

A schematic of a gated fiber detector (GFD) is shown in Fig. 1. The GFD has the following main parts, a reflective layer, a scintillating layer, a vacuum sealing glass window, a photon-coupling cone, a fiber, and a gated photomultiplier tube (gPMT).

\subsection{Reflective layer}

The reflective layer has two functions. First, it reflects the scintillating light, and then highly improves the lightcollecting efficiency. Second, it can reflect the original laser to protect electronics and PMT followed. Wavelengths of HILs today are normally in the range 200-2000 $\mathrm{nm}$ with intensities up to $10^{22} \mathrm{~W} / \mathrm{cm}^{2}$ [27], compared with a typical PMT's working scintillating light intensity of $10^{-9} \mathrm{~W} / \mathrm{cm}^{2}$, or in other words, detecting a single photon. If a small amount of the original photons from the main laser enters the PMT, it can cause the PMT to be blind, which may require a long recovery time, or make the PMT totally damaged in the worst case. Even after diffuse reflecting inside a laser target chamber, the straggling light intensity may be reduced by several orders of magnitude. However, it is still too strong for a PMT to accept it directly and can kill the PMT easily.

A thin layer of aluminum, polytetrafluoroethylene (PTFE), or barium sulfate $\left(\mathrm{BaSO}_{4}\right)$ can be used for this purpose [28-31]. With a few $\mu \mathrm{m} \mathrm{Al}$, charged particles can relatively easily pass through. For light with a wavelength in the range of $>200 \mathrm{~nm}$, a $20 \mu \mathrm{m}$ layer of $\mathrm{Al}$ can reduce the original incoming laser intensity by more than $10^{-6}$ times. At the same time, this layer can reflect the scintillating light with an efficiency of approximately $80 \%-99 \%$ $[28,30]$, which means that the scintillating light-collecting efficiency can be almost doubled.

Al can be oxidized relatively easily if exposed to the atmosphere. The oxidized layer $\left(\mathrm{Al}_{2} \mathrm{O}_{3}\right)$ could be as thick as a few $\mu \mathrm{m}$. The oxidization will not cause problems here due to the following facts. On the one hand, the oxidized layer can also stop the original laser. On the other hand, the surface toward the scintillator is airtight, and this side cannot be oxidized. It can still serve as a mirror to reflect the scintillating light that lasts for a very long time.

A list of reflectors that are frequently used in scintillators is listed in Table 1. As shown in the table, a reflecting coefficient of 0.99 is achievable. 


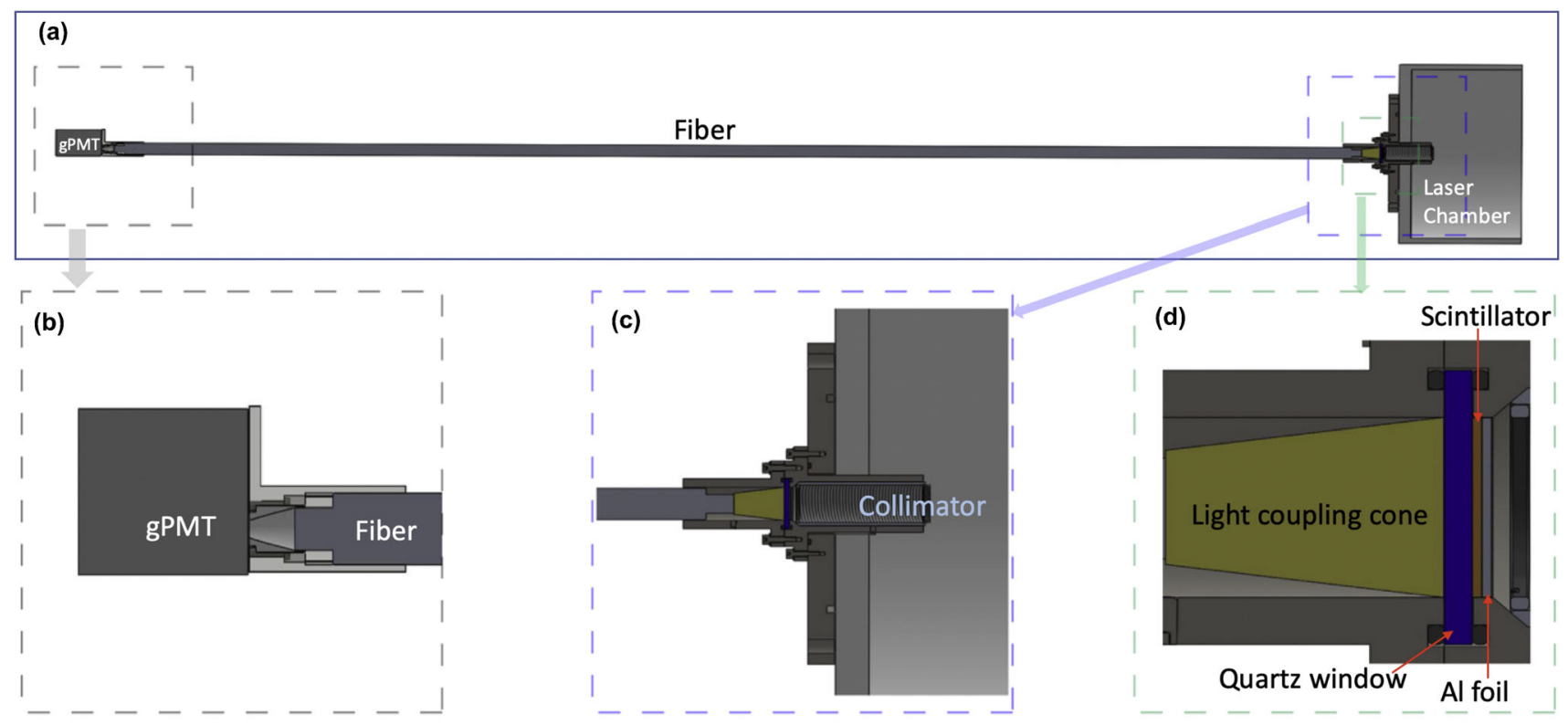

Fig. 1 (Color online) Schematic drawing of a gated fiber detector (GFD). $\mathbf{a}$ is the full view of the GFD, and $\mathbf{b}-\mathbf{d}$ are the zoom-in structures of different parts. As shown in (c) and (d), it has a reflecting $\mathrm{Al}$ foil layer, a scintillating layer, a quartz glass window for sealing vacuum, and a photon-coupling cone for collecting scintillator photons. The length of the fiber is adjustable, which can transport scintillating photons to a desired distance, and then avoid strong EMPs in the target area. The gPMT can be turned off at the moment when the EMPs arrive to further reduce the EMP effects
Table 1 A list of reflectors commonly used for scintillating experiments

\begin{tabular}{llll}
\hline Reflector & Refl. Coeff. (@ $440 \mathrm{~nm})$ & Thickness $(\mathrm{mm})$ & Reference \\
\hline PTFE tape & 0.99 & 0.06 & {$[28,29]$} \\
Magnesium oxide & 0.98 & 1 & {$[32]$} \\
Barium sulfate & 0.98 & 0.12 & {$[31,32]$} \\
Titanium dioxide paint & 0.95 & 0.14 & {$[29]$} \\
Aluminum foil & 0.79 & 0.025 & {$[28]$} \\
Tyvek paper & 0.98 & 0.11 & {$[28]$} \\
\hline
\end{tabular}

\subsection{Scintillator layer}

For different physical purposes, one can choose different materials for the scintillating layer. For HIL applications, normally a fast rising time is required. Therefore, we prefer fast response scintillators like plastic, as well as inorganic ones including LYSO $\left((\mathrm{LuY})_{2} \mathrm{SiO}_{5}: \mathrm{Ce}\right)$, YAP $\left(\mathrm{YAlO}_{3}\right)$, YAG $\left(\mathrm{Y}_{3} \mathrm{Al}_{5} \mathrm{O}_{3}\right)$, and $\mathrm{LSO}\left(\mathrm{Lu}_{2} \mathrm{SiO}_{5}: \mathrm{Ce}\right)$, etc. [30]. The properties of them are listed in Table 2.

If there has a high neutron flux in the background, inorganic scintillators are preferred. Organic scintillators normally have a high percentage of hydrogen inside. Neutrons have a very high scattering cross section on hydrogen, which results in high background noise.

If using GFD to detect charged particles, a vacuum is necessary. Therefore, after the scintillate layer, a quartz glass window is employed to separate the target chamber vacuum from the atmosphere.
Hygroscopic scintillating materials are not convenient to be used. They have to be sealed completely to prevent them from catching moisture in the air. When used for detecting charged particles, the sealing materials would be a dead layer causing an extra measurement uncertainty. Therefore, non- or low- hygroscopic scintillators are preferred. The hygroscopic property of different scintillators is also listed in the Table 2.

\subsection{Fiber coupling}

In a typical HIL experiment, EMPs are very strong near the targets. With an increase in the distance to the target, the EMP becomes weaker, roughly following inverse square law. The closer the detectors and electronics to the target, the stronger the EMP they will suffer. Furthermore, because of the limited space, electronics cannot be shielded fully to avoid impacts from EMPs. Therefore, by using an optical fiber to transfer the scintillating light to a distant 
Table 2 Comparison of major parameters of different type scintillators which have relatively fast light decay times

\begin{tabular}{lllllllll}
\hline Properties & LYSO & LSO & Plastic (EJ200) & YAP:Ce & YAG:Ce & PWO & CsI & LaBr 3 :Ce \\
\hline Density (g/cm $\left.{ }^{2}\right)$ & 7.1 & 7.35 & 4.51 & 5.37 & 4.57 & 8.3 & 4.51 & 5.29 \\
Photon yield (photons/MeV) & 33200 & 30000 & 10000 & 10000 & 8000 & $120 / 40$ & $34000 / 18000$ & 52000 \\
Decay time (ns) & 36 & 40 & 2.1 & 25 & 70 & $30 / 10$ & $30 / 6$ & 20 \\
Peak wavelength (nm) & 420 & 420 & 425 & 370 & 550 & $425 / 420$ & $420 / 310$ \\
Refractive index & 1.81 & 1.82 & 1.58 & 1.95 & 1.82 & 2.20 & 1.79 & 356 \\
Hygroscopic & No & No & No & No & No & No & Slight & Yes \\
\hline
\end{tabular}

location from the target, EMPs can be reduced, together with better shielding for electronics with more materials. One expects a much smaller background noise by using fiber.

When choosing an optical fiber, the main factors are its transmission attenuation at different wavelengths and the numerical aperture (NA). As shown in Table 2, wavelengths of scintillators for general purposes are in the UV region of approximately 300-500 $\mathrm{nm}$. Therefore, UV fibers made from quartz or liquid can be used. For a fiber which has a specific NA, only scintillating light with an incident angle $\theta[33]$,

$\theta \leq \arcsin \left(\mathrm{NA} \cdot \frac{n_{1}}{n_{2}}\right) \equiv \theta_{\max }$

can pass through it, where $n_{1} / n_{2}$ is the refractive index of the light cone and fiber, respectively.

For quartz fibers, the larger the diameter, the harder it is to bend. Therefore, quartz fibers with diameters larger than $1.5 \mathrm{~mm}$ are hardly found in markets. Liquid UV fibers can be made with diameters greater than $10 \mathrm{~mm}$. However, the transmission attenuation of liquid UV fibers is normally larger than that of quartz UV fibers. A typical liquid UV fiber has an attenuation of $0.4 \mathrm{~dB} / \mathrm{m}$ at $400 \mathrm{~nm}$, compared with that of quartz, $0.05 \mathrm{~dB} / \mathrm{m}$.

\subsection{Light-coupling cone}

It can be proved that lens coupling does not have a higher efficiency than the end-to-end coupling method. In fact, if a lens is used to focus the scintillating light onto the ends of a fiber, even the light intensity on the fiber's ends increases, the angle spreading increases at the same time. Therefore, the light-collecting efficiency does not increase at all, because fibers can only accept light with an incident angle smaller than $\theta_{\max }$ in Eq. (1), Therefore, we designed a light-coupling cone, rather than a lens, to improve the light-collecting efficiency.

Materials like quartz or polymethylmethacrylate (PMMA) can be used to make the cones for their low attenuation in the ultraviolet range. The structure of the light-coupling cone is illustrated in Fig. 1d. At the scintillator end, because of the reflective layer which is described in the previous subsection, the light-collecting efficiency is almost doubled. In addition, the cone's side surface is painted to reflect the scintillating light. Painting materials such as EJ-510, Al, Ag, and $\mathrm{BaSO}_{4}$ can be used because of their reflection coefficients in the wavelength range of $300-600 \mathrm{~nm}$.

\subsection{Gated PMT}

To overcome the strong EMPs caused by original main laser pulses, as well as other possible laser-related backgrounds, such as laser-induced neutrons, a gated PMT detector will be used.

A gPMT is a photomultiplier tube with a gating circuit. The "gate" here is different from normal detector gates. Normally detectors' output signals are gated. Thus, one can choose to use or not use an output signal, but the detector itself is always on and working. If very strong EMPs coming, only gating outputs does not help. While here it is designed to be that the detector itself, specifically the bias voltage of the PMT's dynodes, is gated to be power on or power off. Once an EMP coming, the bias of the PMT is turned off, and this will protect the detector as well as the following electronics components from impact or even damage by the EMP.

As shown in Fig. 2, we used a circuit that can close the PMT's bias voltage in $8 \mathrm{~ns}$, and turn it on to a working condition in $70 \mathrm{~ns}$. The time window that keeps the detector working, $T_{\mathrm{W}}$, is $100 \mathrm{~ns}<T_{\mathrm{W}}<\infty$. In typical HIL experiments, EMPs may last from a few ns to tens of ns. Therefore, the gated PMT here can provide protection to detecting electronics.

The time evolution of the EMPs and massive particle signals to be detected are shown in Fig. 3. As shown there, EMPs and massive reaction products to be detected are generated at time $T=0$. Because the massive particles have a slower speed than the EMPs, they arrive at gPMT 


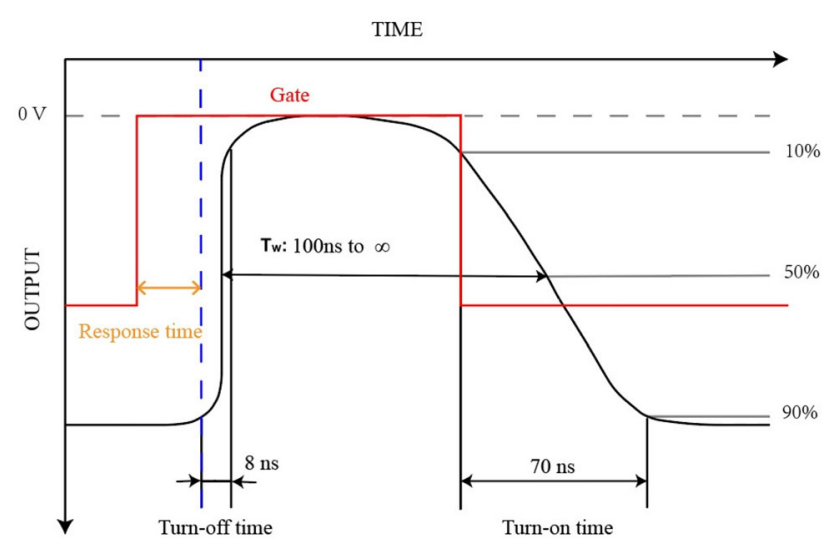

Fig. 2 (Color online) Schematic of the timing sequence of the gPMT, which works in the normal on model. This gPMT can respond to a gate signal quickly. Specifically, it can turn off the power supply to the PMT in $8 \mathrm{~ns}$ and turn it on in $70 \mathrm{~ns}$. The time window $T_{\mathrm{W}}$ can be tuned in the range of $100 \mathrm{~ns}<T_{\mathrm{W}}<\infty$ by the width of the gate signal. In this normal on model, the PMT is shut down in the time window $T_{\mathrm{W}}$

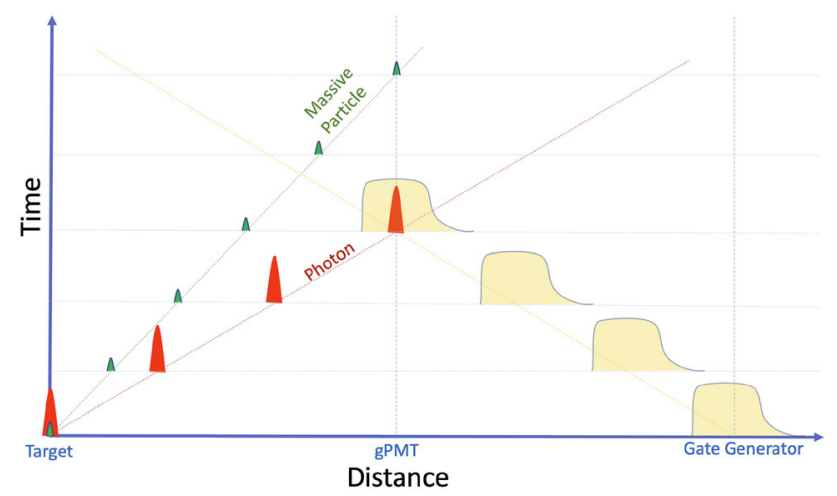

Fig. 3 (Color online) Schematic space-time drawing of photons and massive particles traveling after lasers bombard a target. At time $t=0$, both photons and massive particles are generated simultaneously. The photons move at a speed of $c$ (red line), while the massive particles move at a lower speed (green line). By sending a gate signal (the yellow line) to the gPMT, photon signals from the EMP can be vetoed, while the massive particle signals can be recorded by the gPMT

position earlier than the massive particles. Therefore, by sending a gate signal from a gate generator, one can make the gPMT not respond to the EMP signals, but respond to the massive particle signals.

The massive particles here could be neutrons or charged particles. In fact, besides massive particles generated at around $T=0$, any particles, including photons, which have different TOF, can be detected in this setup. For example, photons emitted from excited nuclei, that is, nuclear isomers, have a longer TOF than the original EMP. Therefore, this type of photons, as well as other massive particles that are generated at a larger time $T$ of course, can be recorded in this manner.

\section{Simulation of scintillating photon collection}

A numerical simulation was carried out to optimize the light-collecting efficiency of the cone. A home-written programmer based on the ROOT is used. From the scintillator, a random scintillating ray was generated, which then travels inside the light-collecting cone with a diffused surface. At $i$ time, the ray hits the cone's surface or reflective mirror layer, the corresponding reflection coefficient $R_{i}$ is recorded. Once the ray hits the exit window, depending on the angle $\theta$ between the ray and the surface of the exit window, the value $I=I_{0} \prod_{i} R_{i}$ is recorded as the light intensity that passes through the fiber if $\theta \leq \sin (\mathrm{NA})$, or discarded if $\theta>\sin (\mathrm{NA})$, where $I_{0}$ is the intensity of the original scintillating ray. The following simulation input parameters are assumed: reflection coefficient of the side painting $R_{\mathrm{p}}=98 \%$; reflection coefficient of the reflective mirror layer $R_{\mathrm{m}}=98 \%$; the scintillator diameter (entrance window) $D_{\mathrm{S}}=10 \mathrm{~mm}$; the fiber (exit window) diameter $1 \leq d_{\mathrm{e}} \leq 15 \mathrm{~mm}$; and isotropic emitting angle of the scintillating photons. The light-coupling efficiency $\eta$ as a function of $D_{\mathrm{e}}$ and the $N A$ is shown in Fig. 4. One can see that $\eta$ is high at $D_{\mathrm{e}}=8 \mathrm{~mm}$. Only liquid fibers are available with such high $D_{\mathrm{e}}$. Quartz fibers with $D_{\mathrm{e}}=1.5 \mathrm{~mm}$ and NA $=0.5$ are available, and the coupling efficiency $\eta>1 \%$ can be expected by using them.

In Table 3, light-collecting efficiencies for different core diameters and NAs are listed. As shown there, with a smaller diameter, light-collecting efficiency reduces quickly as diameter dropping. In fact, according to the second thermodynamic law, the percentage of the photons which has incident angle $\theta<\theta_{\max }$ keeps as a constant

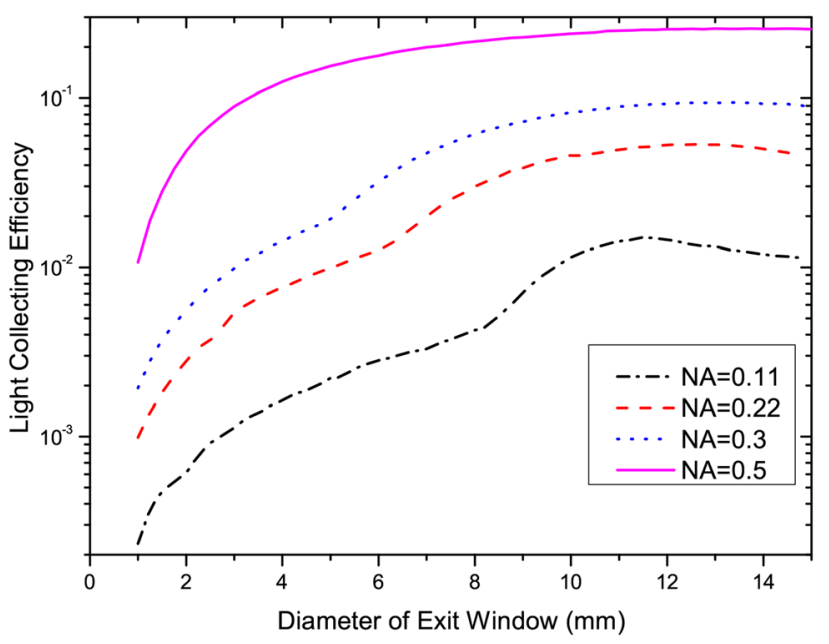

Fig. 4 (Color online) Simulation results of light-collecting efficiency by a cone with an entrance window diameter of $10 \mathrm{~mm}$, a height of 8 $\mathrm{mm}$, and a variable exit window diameter. The cone was coupled to fibers with NAs of $0.11,0.22,0.3$, and 0.5 . The reflective efficiency is set to be $98 \%$ 
Table 3 Relationship between NAs of fibers and light collection efficiencies

\begin{tabular}{llll}
\hline NA & $\theta_{\max }$ & $\eta_{\operatorname{sim}}^{(1)}\left(D_{\mathrm{e}}=8.0 \mathrm{~mm}\right)^{\mathrm{a}}$ & $\eta_{\operatorname{sim}}^{(2)}\left(D_{\mathrm{e}}=1.5 \mathrm{~mm}\right)^{\mathrm{b}}$ \\
\hline 0.11 & $6.3^{\circ}$ & $4.2 \times 10^{-3}\left[4.6 \times 10^{-3}\right]$ & $4.5 \times 10^{-4}\left[1.0 \times 10^{-3}\right]$ \\
0.22 & $12.7^{\circ}$ & $2.7 \times 10^{-2}\left[3.1 \times 10^{-2}\right]$ & $1.8 \times 10^{-3}\left[4.5 \times 10^{-3}\right]$ \\
0.30 & $17.5^{\circ}$ & $6.1 \times 10^{-2}\left[6.9 \times 10^{-2}\right]$ & $3.7 \times 10^{-3}\left[8.8 \times 10^{-3}\right]$ \\
0.50 & $30.0^{\circ}$ & $2.2 \times 10^{-1}\left[2.4 \times 10^{-1}\right]$ & $2.8 \times 10^{-2}\left[7.2 \times 10^{-2}\right]$ \\
\hline
\end{tabular}

a Simulation inputs: $r=98 \%$ and $r=100 \%$ (in brackets)

${ }^{\mathrm{b}}$ Simulation inputs: $r=98 \%$ and $r=100 \%$ (in brackets)

$\theta_{\max }$ is the maximum light cone angle at which light can pass through the fiber, which has the corresponding NA. $\eta_{\text {sim }}$ is the simulated light-collecting efficiency which has assumptions including reflecting efficiency $r=98 \%$ (and perfect $r=100 \%$ also shown in brackets), the diameter of coupling light cone entrance window 10 $\mathrm{mm}$, length of $8 \mathrm{~mm}$, and an existing window diameter $D_{\mathrm{e}}=1.5,8$ $\mathrm{mm}$

whatever shape of the reflection surfaces are. Therefore, if the reflecting efficiency of the surfaces is $100 \%$, the collecting efficiency will remain constant as well, regardless of the size of the existing window's diameter. It is the reflected times $N$ that reduce the collecting efficiency dramatically. This is because of the collecting efficiency $\eta \propto r^{N}$. Even if $r$ is close to $1, r=0.98$, the power $r^{N}$ decreases rapidly. Clearly, the smaller $N$, the higher the collecting efficiency $\eta \propto r^{N}$. With rough surfaces and diffusing reflection, a ray goes randomly, which results,

$N=\frac{A_{\text {tot }}}{A_{\mathrm{ex}}}$,

where $A_{\text {tot }}$ is the area of the light-coupling cone's outside surface and $A_{\mathrm{ex}}$ is the area of the existing window. From the Eq. 2 one can find that $N$ could be very large! A mirror surface together with a carefully designed geometry may be helpful.

To optimize the light-collecting efficiency $\eta$, the dependence of the cone length $L$ was also studied, as shown in Fig. 5. When $L \rightarrow 0$, the light entrance window touches the existing window, and then

$\lim _{L \rightarrow 0} \eta \propto \frac{A_{\text {ex }}}{A_{\text {in }}}$,

where $A_{\text {in }}$ is the area of incoming window. When $L$ increases, an increasing number of scintillating photons have a higher chance of reaching the exit window with angles close to $\frac{\pi}{2}$, which results in a higher $\eta$. However, the longer the $L$, the more times of reflection. Because of not perfect reflection efficiency, an increasing number of photons will be lost during their path to the existing window, and then result in $\eta$ drops. This effect can be found in

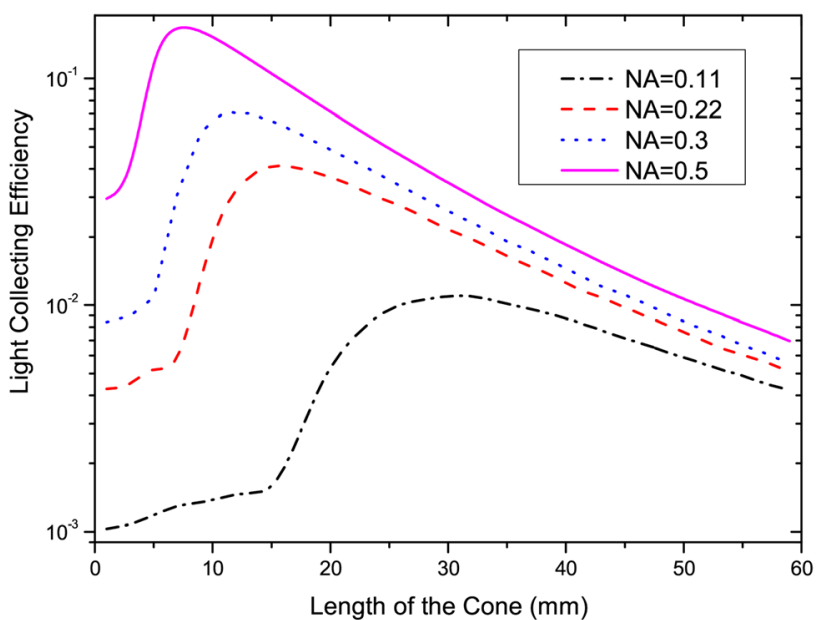

Fig. 5 (Color online) Simulation results of light-collecting efficiency by a cone with an entrance diameter of $5 \mathrm{~mm}$, an exit window diameter of $1.5 \mathrm{~mm}$, and a variable length. The cone was coupled to fibers with NAs of $0.11,0.22,0.3$, and 0.5 . The reflective efficiency is set to be $98 \%$

Fig. 5. For different NA fibers, there is an optimized length. For NA $=0.5, \eta$ has a peak value of approximately $L=6 \mathrm{~mm}$.

\section{Testing on a HIL beam line}

The GFD was tested on a Xing Guang III (XG-III) laser facility located at the Science and Technology on Plasma Physics Laboratory, the Laser Fusion Research Center, Sichuan Province, China. The facility has three laser beams with different wavelengths and duration, fs beam (pulse width $26 \mathrm{fs}$; maximum energy $20 \mathrm{~J}$; wavelength $800 \mathrm{~nm}$ ), ps beam $(0.5-10 \mathrm{ps} ; 370 \mathrm{~J} ; 1053 \mathrm{~nm})$, and ns beam (1.1 ns; $570 \mathrm{~J}$; and $527 \mathrm{~nm}$ ).

In our test runs, only a ps beam was used. The typical energy of the ps beam is about $100 \mathrm{~J}$. The laser beam bombarded a gas jet. The GFD was set at a forward angle of approximately $30^{\circ}$ to the laser beam direction. A plastic scintillator with a thickness of about $3 \mathrm{~mm}$ was used. A collimator with a diameter of $20 \mathrm{~mm}$ was aligned before the GFD. Electrons and ions induced by the laser and gas target interaction were then detected by the GFD. and the signals were recorded by a $200 \mathrm{MHz}$ oscilloscope. The oscilloscope was triggered by a signal synchronous with the laser beam. The same trigger signal was also used as the gate for the GFD. By tuning the relative time between the oscilloscope starting to record and the laser striking the target, we could turn off/on the GFD. 

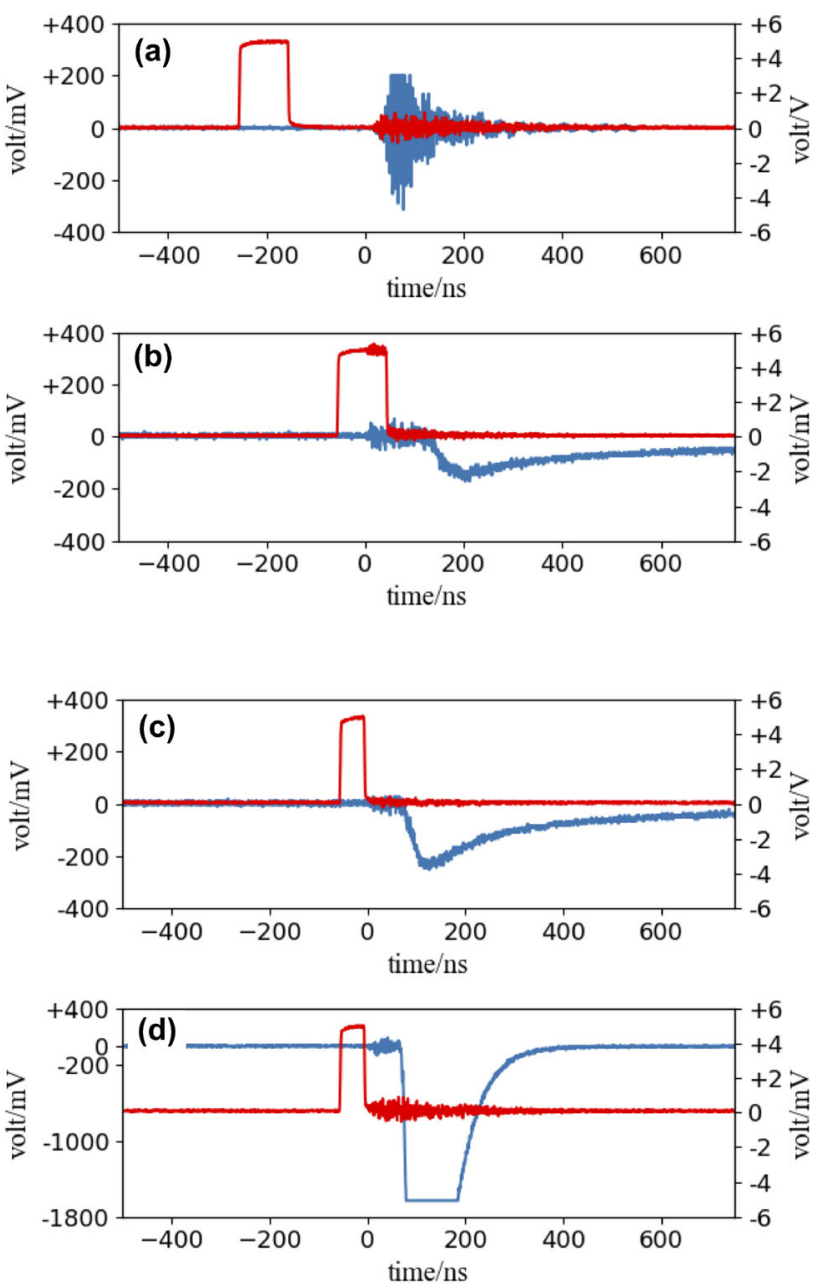

Fig. 6 (Color online) Online test results at XG-III laser facility. The square waves (red) are the trigger for the gPMT, and the blue curves are signals from a gPMT. The gPMT is in normal on mode. The delay time of the trigger was $-260 \mathrm{~ns}$ for (a) and $-60 \mathrm{~ns}$ for (b), (c), and (d). The pulse was turned to $100 \mathrm{~ns}$ for (a) and (b) and $50 \mathrm{~ns}$ for (c) and (d). In a, the power of the gPMT was turned off, whereas in others, the power was on. The gPMT gain in (d) was approximately 10 times higher than that in (b) and (c). Look at the context for details

The results are shown in Fig. 6a represents "totally" turning off the GFD. For "totally," we mean that the power supply for the GFD is unplugged, whereas, in the gating model, the power supply is plugged, and the PMT may not be biased when responding to a gate signal, while other electronics of the GFD were still working. However, in the "totally turn off" case, the electronics were not working. From Fig. 6a, there is a large signal. Its positive and negative amplitudes are almost equal. It should be mentioned that the signal peak at approximately $50 \mathrm{~ns}$ in the spectrum is due to circuit response. The original EMPs were very narrow ( $<1 \mathrm{~ns}$ ) and quick (approximately $12 \mathrm{~ns}$ after laser-target interaction). In fact, the GFD was located only about $4 \mathrm{~m}$ from the target, and it took only $12 \mathrm{~ns}$ for photons to travel from the target to there.
By tuning the delay, we gated out the EMPs, as shown in Fig. 6 b. One can see that there is a delay of approximately $70 \mathrm{~ns}$ between the gate signal and the measured signal. This is due to the gPMT response time is about $70 \mathrm{~ns}$. The measured signals correspond to energetic electrons and ions from the laser-induced plasmablasts. In Fig. $6 c$, by tuning the width of the gate signal from $100 \mathrm{~ns}$ to $50 \mathrm{~ns}$, the EMP can still be suppressed. In this way, one can reduce the GFD response time. Of course, there is a risk of damaging the PMT if response time is reduced too much. Keeping the same gate signal, and increasing the gPMT gain 6 times larger, the resulting spectrum is shown in Fig. 6d. The signal was saturated at about 70-195 ns.

Based on the online test results shown in Fig. 6, the prototype GFD developed by us works as expected. In this proof-of-principle test, the signals were induced by the plasma, which was composed of electrons and ions. Particles could be identified yet with the current setup. However, it can be easily improved in a real physical measurement. Together with traditional particle identification methods such as $\triangle E-E, T O F, m / q$, etc., energy and time signals from the GFD will help identify the type of particles. For example, by replacing the image plates that are currently used in Thomson spectrometer focus planes $[24,25]$ in typical HIL experiments with GFDs, one can have extra time information, as well as an improved energy signal compared with that from the image plate, and then obtain much better particle identification capabilities. In addition to changed particles, this GFD can also be used to detect neutrons in harsh environments with a relative neutron-sensitive scintillator.

Furthermore, photons may be emitted after time zero from excited nuclei and atoms that have relatively long metastable (or isomer for nuclei) states. As shown in Fig. 3, if these photons arrive at the GFD away from the main peak, they can also be detected. With the capability of working in strong EMPs, the GFD will benefit HIL experiments by measuring neutrons, photons, and charged particles.

\section{Summary}

A major obstacle in laser nuclear physics studies is how to distinguish weak nuclear reaction product signals from very strong EMP signals induced by HILs. To overcome this difficulty, a gated fiber detector for HIL applications has been developed. By using reflective foil, fiber, and gated PMT, strong EMPs which cause dysfunction of electronics in HIL environments are avoided. By numerical simulation, the parameters like NA are optimized. An online test shows that this prototype GFD can suppress EMP signals efficiently and can be used in HIL 
environments. The GFD can respond approximately $70 \mathrm{~ns}$ after the laser shot on the target, which makes it a good TOF detector to detect massive particles induced by HILs, as well as delayed gamma from excited states of nuclei in HIL-target interactions.

Acknowledgements We would like to acknowledge XG-III staff for operating the laser facility.

Open Access This article is licensed under a Creative Commons Attribution 4.0 International License, which permits use, sharing, adaptation, distribution and reproduction in any medium or format, as long as you give appropriate credit to the original author(s) and the source, provide a link to the Creative Commons licence, and indicate if changes were made. The images or other third party material in this article are included in the article's Creative Commons licence, unless indicated otherwise in a credit line to the material. If material is not included in the article's Creative Commons licence and your intended use is not permitted by statutory regulation or exceeds the permitted use, you will need to obtain permission directly from the copyright holder. To view a copy of this licence, visit http://creativecommons. org/licenses/by/4.0/.

Author Contributions All authors contributed to the study conception and design. Material preparation, data collection and analysis were performed by Po Hu, Zhi-Guo Ma, Zhao Kai, Guo-Qiang Zhang, and Chang-Bo Fu. The first draft of the manuscript was written by Chang-Bo Fu and all authors commented on previous versions of the manuscript. All authors read and approved the final manuscript.

\section{References}

1. T. Ditmire, J.W.G. Tisch, E. Springate et al., High-energy ions produced in explosions of superheated atomic clusters. Nature 386, 54-56 (1997). https://doi.org/10.1038/386054a0

2. T. Tajima, J.M. Dawson, Laser electron accelerator. Phys. Rev. Lett. 43, 267 (1979). https://doi.org/10.1103/PhysRevLett.43.267

3. C.B. Fu, J. Bao, L.M. Chen et al., Laser-driven plasma collider for nuclear studies. Sci. Bull. 60, 1211-1213 (2015). https://doi. org/10.1007/s11434-015-0821-0

4. D. Klir, J. Krasa, J. Cikhardt et al., Efficient neutron production from sub-nanosecond laser pulse accelerating deuterons on target front side. Phys. Plasmas 22, 093117 (2015). https://doi.org/10. $1063 / 1.4931460$

5. D.L. Balabanski, R. Popescu, D. Stutman et al., New light in nuclear physics: the extreme light infrastructure. Europhys. Lett. 117, 28001 (2017). https://doi.org/10.1209/0295-5075/117/28001

6. X. Zhang, J. Zhao, D. Yuan et al., Deuteron-deuteron fusion in laser-driven counter-streaming collisionless plasmas. Phys. Rev. C 96, 055801 (2017). https://doi.org/10.1103/PhysRevC.96. 055801

7. Y.-X. Geng, Q. Liao, Y.-R. Shou et al., Generating proton beams exceeding $10 \mathrm{MeV}$ using high contrast $60 \mathrm{TW}$ laser. Chin. Phys. Lett. 35, 092901 (2018). https://doi.org/10.1088/0256-307x/35/9/ 092901

8. Z.G. Ma, H.Y. Lan, W.Y. Liu et al., Photonuclear production of medical isotopes $62,64 \mathrm{Cu}$ using intense laser-plasma electron source. Matter Radiat. Extremes 4, 064401 (2019). https://doi. org/10.1063/1.5100925

9. W. Luo, W.Y. Liu, T. Yuan et al., QED cascade saturation in extreme high fields. Sci. Rep. 8, 8400 (2018). https://doi.org/10. 1038/s41598-018-26785-8
10. X.L. Wang, Z.Y. Xu, W. Luo et al., Transmutation prospect of long-lived nuclear waste induced by high-charge electron beam from laser plasma accelerator. Phys. Plasmas 24, 093105 (2017). https://doi.org/10.1063/1.4998470

11. J. Nuckolls, L. Wood, A. Thiessen et al., Laser compression of matter to super-high densities: thermonuclear (CTR) applications. Nature 239, 139-142 (1972). https://doi.org/10.1038/239139a0

12. J. Zweiback, R.A. Smith, T.E. Cowan et al., Nuclear fusion driven by coulomb explosions of large deuterium clusters. Phys. Rev. Lett. 84, 2634 (2000). https://doi.org/10.1103/PhysRevLett. 84.2634

13. L. Torrisi, S. Cavallaro, M. Cutroneo et al., Deuterium-deuterium nuclear reaction induced by high intensity laser pulses. Appl. Surf. Sci. 272, 42-45 (2013). https://doi.org/10.1016/j.apsusc. 2012.02.077

14. V.Y. Bychenkov, V.T. Tikhonchuk, S.V. Tolokon-nikov, Nuclear reactions triggered by laser-accelerated high-energy ions. J. Exp. Theor. Phys. 88, 1137-1142 (1999). https://doi.org/10.1134/1. 558902

15. S. Tavernier, Experimental Techniques in Nuclear and Particle Physics (Springer Science \& Business Media, NewYork, 2010)

16. Y.-F. He, X.-F. Xi, S.-L. Guo et al., Calibration of CR-39 solidstate track detectors for study of laser-driven nuclear reactions. Nucl. Sci. Tech. 31, 42 (2020). https://doi.org/10.1007/s41365020-0749-1

17. M.-H. Wang, J.-L. Qu, M. Zhu, Partially overlapped dual laser beams to reduce ablation craters. Chin. Phys. Lett. 37, 015202 (2020). https://doi.org/10.1088/0256-307X/37/1/015202

18. S.-K. He, J.-L. Jiao, Z.-G. Deng et al., Generation of ultrahighvelocity collisionless electrostatic shocks using an ultra-intense laser pulse interacting with foil-gas target. Chin. Phys. Lett. 36, 105201 (2019). https://doi.org/10.1088/0256-307x/36/10/105201

19. F. Consoli, V.T. Tikhonchuk, M. Bardon et al., Laser produced electromagnetic pulses: generation, detection and mitigation. High Power Laser Sci Eng 8, e22 (2020). https://doi.org/10.1017/ hpl.2020.13

20. T.W. Jeong, P.K. Singh, C. Scullion et al., CR-39 track detector for multi-MeV ion spectroscopy. Sci. Rep. 7, 2152 (2017). https:// doi.org/10.1038/s41598-017-02331-w

21. Y. Zhang, H.-W. Wang, Y.-G. Ma et al., Energy calibration of a CR-39 nuclear-track detector irradiated by charged particles. Nucl. Sci. Tech. 30, 87 (2019). https://doi.org/10.1007/s41365019-0619-x

22. Y. Zhang, L.-X. Liu, H.-W. Wang et al., Primary yields of protons measured using CR-39 in laser-induced deuteron-deuteron fusion reactions. Nucl. Sci. Tech. 31, 62 (2020). https://doi.org/ 10.1007/s41365-020-00769-8

23. E. Awad, M. Rana, M. Al-Jubbori, Bulk etch rates of CR-39 at high etchant concentrations: diffusion-limited etching. Nucl. Sci. Tech. 31, 118 (2020). https://doi.org/10.1007/s41365-020-008306

24. L. Zhao, Z.-J. Chen, H.-B. Sang et al., Spatial characteristics of thomson scattering spectra in laser and magnetic fields. Chin. Phys. Lett. 36, 074101 (2019). https://doi.org/10.1088/0256307X/36/7/074101

25. G. Di. Giorgio, F. Consoli, R. De. Angelis et al., Development of advanced Thomson spectrometers for nuclear fusion experiments initiated by laser. J. Instrum. 15, C10013 (2020). https://doi.org/ 10.1088/1748-0221/15/10/C10013

26. C. Fu, Q. Tang, D. Fang et al., Proton energy detectors for environments of high intensity electromagnetic pulses. Patent application in process under No. CN112099072A, 2020

27. T. Tajima, G. Mourou, Zettawatt-exawatt lasers and their applications in ultrastrong-field physics. Phys. Rev. Spec. Top. Accel. Beams 5, 031301 (2002). https://doi.org/10.1103/PhysRevSTAB. 5.031301 
28. M. Janecek, W. Moses, Optical reflectance measurements for commonly used reflectors. IEEE Trans. Nucl. Sci. 55, 2432-2437 (2008). https://doi.org/10.1109/TNS.2008.2001408

29. M. Janecek, Reflectivity spectra for commonly used reflectors. IEEE Trans. Nucl. Sci. 59, 490-497 (2012). https://doi.org/10. 1109/TNS.2012.2183385

30. M.N. Ullah, E. Pratiwi, J.H. Park et al., Studies on sub-millimeter LYSO:Ce, Ce:GAGG, and a new Ce:GFAG block detector for PET using digital silicon photomultiplier. Nucl. Instrum. Methods A 911, 115-122 (2018). https://doi.org/10.1016/j.nima.2018. 09.029
31. J.B. Schutt, J.F. Arens, C.M. Shai et al., Highly reflecting stable white paint for the detection of ultraviolet and visible radiations. Appl. Opt. 13, 2218 (1974). https://doi.org/10.1364/ AO.13.002218

32. F. Grum, G.W. Luckey, Optical sphere paint and a working standard of reflectance. Appl. Opt. 7, 2289 (1968). https://doi.org/ 10.1364/AO.7.002289

33. J. Elsey, D.R. McKenzie, J. Lambert et al., Optimal coupling of light from a cylindrical scintillator into an optical fiber. Appl. Opt. 46, 397 (2007). https://doi.org/10.1364/AO.46.000397 